\title{
Inhibition of lipogenesis and induction of apoptosis by valproic acid in prostate cancer cells via the C/EBPa/SREBP-1 pathway
}

\section{Bo Pang}

Tianjin Medical University

Juanjuan Zhang

Tianjin Medical University

Jihong Yuan

Tianjin Medical University

Yanan Shi

Tianjin Medical University

Ling Qiao ( $\nabla$ qiaoling@tijmu.edu.cn )

Tianjin Medical University https://orcid.org/0000-0003-1160-9738

\section{Research}

Keywords: Valproic acid, Prostate cancer, Lipogenesis, Apoptosis, CCAAT/enhancer-binding protein a, Sterol regulatory element-binding protein-1

Posted Date: January 10th, 2020

DOI: https://doi.org/10.21203/rs.2.20628/v1

License: (c) (i) This work is licensed under a Creative Commons Attribution 4.0 International License. Read Full License 


\section{Abstract}

\section{Background}

Lipid metabolism reprogramming is now accepted as a new hallmark of cancer. Hence, targeting the lipogenesis pathway may be a promising avenue for cancer treatment. Valproic acid (VPA) emerges as a promising drug for cancer therapy, however, the underlying mechanisms are not yet fully understood. This study aimed to investigate the effects and mechanism of VPA on cell viability, lipogenesis, and apoptosis in human prostate cancer PC-3 cells.

\section{Methods}

The effects of VPA on the viability of PC-3 cells were investigated using MTT assay and wound-healing assay. Oil Red $O$ staining was used to examine lipid droplets, and DAPI staining assay and Annexin V-PI staining assay were used to measure the extent of cell apoptosis. Quantitative real-time PCR and Western blotting were used to determine the expression of lipogenesis and apoptosis genes. Statistical and analytical data with SPSS 17.0 Software, statistical significance was set at ${ }^{*} P<0.05, * \star P<0.01$, and $* \star * P$ $<0.001$ levels.

\section{Results}

The results showed that VPA significantly reduced lipid accumulation and induced apoptosis of PC-3 cells. Moreover, the expression of CCAAT/enhancer-binding protein a (C/EBPa), as well as sterol regulatory element-binding protein 1 (SREBP-1) and its downstream effectors, including fatty acid synthase (FASN), acetyl CoA carboxylase 1 (ACC1), and antiapoptotic B cell lymphoma 2 (Bcl-2), markedly decreased in PC-3 cells after VPA administration. Mechanistically, the overexpression of C/EBPa rescued the levels of SREBP-1, FASN, ACC1, and Bcl-2, enhanced lipid accumulation and attenuated apoptosis of VPA-treated PC-3 cells. Conversely, C/EBPa knockdown by siRNA further decreased the levels of SREBP-1, FASN, ACC1, and Bcl-2 and lipid accumulation and enhanced apoptosis. In addition, SREBP-1a and 1c enhanced the expression of FASN and ACC1, but only SREBP-1a had a significant effect on Bcl-2 expression in VPA-treated PC-3 cells.

\section{Conclusions}

On the whole, it is concluded that VPA significantly inhibits cell viability via inhibiting lipogenesis and inducing apoptosis by the C/EBPa/SREBP-1 pathway in PC-3 cells. Hence, VPA, which targets lipid metabolism and apoptosis, is a promising candidate for PCa chemotherapy.

\section{Background}

Over the past decades, an increase in the incidence of various metabolic disorders, including type 2 diabetes mellitus, obesity, nonalcoholic fatty liver disease, atherosclerosis, thromboembolism, and cancer, has been strongly associated with dysregulated lipid metabolism. Noticeably, the alteration of lipid 
metabolism has been increasingly recognized as a hallmark of cancer cells. Therefore, targeting lipid metabolic reprogramming is a potential cancer treatment strategy $[1,2]$. Prostate cancer (PCa) is a common malignant tumor in the urinary system and the third leading cause of cancer-related death in men worldwide. PCa is well recognized as a lipid-enriched tumor, an important biological feature of PCa progression is the dysregulation of lipid metabolism [3-5]. Previous studies indicated that inhibiting de novo lipogenesis via deleting calcium/calmodulin-dependent protein kinase kinase 2, activating AMPactivated protein kinase, or selectively inhibiting fatty acid synthase (FASN) reduced cell growth in human PCa cells $[6,7]$.

Valproic acid (VPA), a histone deacetylase inhibitor, is known as an anticonvulsant and mood-stabilizing drug for more than 40 years. Previous study indicates that VPA suppresses adipogenesis and decreases the expression levels of peroxisome-proliferator-activated receptor $\gamma$, CCAAT/enhancer-binding protein a (C/EBPa), and FASN, which are key regulators of adipogenesis in adipocytes [8]. Treatment of ob/ob mice with VPA for 14 days results in decreased hepatic fat accumulation [9]. Recent studies have shown that VPA can suppress the malignancy of various cancers, including prostate cancer, glioblastoma, and melanoma, because it inhibits tumor growth and metastasis, induces differentiation and apoptosis, and enhances chemotherapy sensitivity [10-12]. Although study indicates that VPA pretreatment suppresses PCa cell viability [13], its potential roles and, more importantly, the underlying mechanisms of its actions have not been extensively studied.

The present study aimed to gain insights into the antitumor mechanisms of VPA in PCa cells. The results showed that VPA inhibited cell viability in PC-3 cells through suppressing lipid accumulation and inducing cell apoptosis. It involved the key regulators of lipogenesis, including C/EBPa and sterol regulatory element-binding protein-1 (SREBP-1) and their target genes. These findings might provide a therapeutic insight for PCa targeting lipogenesis and apoptosis.

\section{Methods}

Cell culture and pharmacological intervention

The human prostate cancer PC-3 cells (obtained from Tianjin Medical University Cancer Institute and Hospital, National Clinical Research Center of Cancer, Tianjin, China) were cultured in RPMI 1640 medium (Gibco, CA, USA) with 10\% ( $\mathrm{v} / \mathrm{v}$ ) fetal bovine serum (Gibco, CA, USA) containing antibiotics $(100 \mathrm{U} / \mathrm{mL}$ penicillin $\mathrm{G}$ and $100 \mathrm{mg} / \mathrm{mL}$ streptomycin) and incubated at $37^{\circ} \mathrm{C}$ in $5 \% \mathrm{CO}_{2}$ humidified incubator. As per experimental requirements, the PC-3 cells were treated with valproic acid (VPA, purchased from SigmaAldrich Chemicals, MO, USA) and then subjected to 3-(4,5-dimethylthiazol-2-yl)-2,5-diphenyltetrazolium bromide (MTT) cell viability assay, wound-healing assay, Oil-Red 0 staining, 4', 6'-diamidino-2-phenylindole (DAPI) staining assay, Annexin V-propidium iodide (PI) staining assay, quantitative real-time polymerase chain reaction (qRT-PCR), and Western blot analysis.

MTT cell viability assay 
PC-3 cells were seeded in 96-well plates at a density of $2 \times 10^{4}$ cells/well and incubated overnight, followed by treatment with the indicated concentrations of VPA. At the endpoint of treatment, $10 \mu \mathrm{L}$ of $5 \mathrm{mg} / \mathrm{mL}$ MTT in phosphate-buffered saline (PBS, purchased from Amresco Inc., OH, USA) was added to each well and incubated in the dark for $4 \mathrm{~h}$ at $37^{\circ} \mathrm{C}$. The cell culture medium was replaced with $150 \mu \mathrm{L}$ of dimethyl sulfoxide, and the mixture was stirred for $10 \mathrm{~min}$. The optical density (OD) values for samples were measured using a Pan-wavelength microplate reader at $570 \mathrm{~nm}$. Triplicate wells were used for each sample, and the experiments were repeated at least three times to get means and standard deviations. Each test should include a blank containing a complete culture medium without cells.

Wound-healing assay

The cell migration ability was examined using the wound-healing assay. PC-3 cells in each group were seeded into a six-well plate. When the confluence reached $60 \%-80 \%$, a wound was built afterward across the cell monolayer using a BioClean $1000 \mu \mathrm{L}$ plastic pipette tip. The cells were rinsed with PBS three times and incubated with fresh medium containing different dosages of VPA for another 4 days or $2.0 \mathrm{mM}$ VPA for another 2,4 , and 6 days. The cell migration into the wound area was photographed under an inverted microscope (Leica, Germany) with $100 \times$ magnification. The relative migration rate was calculated using the following formula: relative migration rate $=$ (distance between the gap at $0 \mathrm{~h}$ distance between the gap at each time point)/distance between the gap at $0 \mathrm{~h} \times 100 \%$.

\section{Oil-Red 0 staining}

PC-3 cells were seeded in a 6-well plate containing a glass coverslip bottom. The cells attached to the coverslip were fixed in $4 \%$ paraformaldehyde for 15 min and then analyzed with an Oil-Red 0 stain kit (Nanjing Jiancheng Bioengineering Institute, China) according to the supplier's instructions. The lipid droplets stained with Oil-Red O were visualized with an Olympus BX53 microscope (Olympus Corporation, Tokyo, Japan) equipped with a DP72 microscope digital camera and Image-Pro Plus 7.0 software. Light absorbance of the extracted dye was measured at $520 \mathrm{~nm}$.

Western blot analysis

The cultured cells were collected and solubilized using protein lysis buffer. Lysates were cleared by centrifugation, and proteins were separated by gel electrophoresis. The membranes were blocked in PBS containing $0.1 \%$ Tween 20 and $5 \%(\mathrm{w} / \mathrm{v})$ milk for $2 \mathrm{~h}$ at room temperature. The blots were first incubated with primary antibodies: anti-C/EBPa, anti-SREBP-1 (Abcam, Cambridge, UK), anti-Flag (Sigma-Aldrich), anti-FASN, anti-acetyl CoA carboxylase 1 (ACC1), anti-B cell lymphoma 2 (Bcl-2), or anti- $\beta$-Actin (Proteintech, Wuhan, China), followed by incubation with the appropriate secondary antibody, that is, goat anti-rabbit IgG $(\mathrm{H}+\mathrm{L})$-HRP or goat anti-mouse IgG $(\mathrm{H}+\mathrm{L})$-HRP (RayBiotech, Beijing, China). The detection was performed using an enhanced chemiluminescence kit (Advansta, CA, USA).

DAPI staining assay 
PC-3 cells $\left(4 \times 10^{5}\right.$ per well) were cultured in a six-well plate, and nuclear morphology was tested using a DAPI staining assay. Following treatment with VPA $(0,1.0,2.0,5.0$, and $10.0 \mathrm{mM})$ for 4 days, the cells were fixed with $4 \%$ paraformaldehyde for $15 \mathrm{~min}$ and permeabilized with $0.1 \%$ Triton X-100 for 2 min. Then, they were stained with $10 \mu \mathrm{g} / \mathrm{mL}$ DAPI (purchased from Roche Corporation, Switzerland) at $37^{\circ} \mathrm{C}$ in the dark for $10 \mathrm{~min}$. The nuclear morphology was viewed under ultraviolet light, and the images were captured using an inverted fluorescence microscope (Leica, Germany). Apoptotic cells were identified on the basis of characteristic changes, including nuclear condensation, fragmentation, and presence of apoptotic bodies [14].

Annexin V-PI staining assay

The cells $\left(4 \times 10^{5}\right.$ per well) were seeded in a six-well plate overnight and treated with varying concentrations of VPA for 4 days. To measure the extent of cell apoptosis, an Annexin V-fluorescein isothiocyanate (FITC) apoptosis detection kit was used according to the manufacturer's instructions (Sungene Biotech, Tianjin, China). In brief, the cells were harvested and suspended in $1 \times$ binding buffer. Then, $100 \mu \mathrm{L}$ of the cell suspension was incubated with $5 \mu \mathrm{L}$ of Annexin V-FITC for 10 min, followed by incubation with $5 \mu \mathrm{L}$ of PI solution for another $5 \mathrm{~min}$. The labeled cells were then assessed by flow cytometry and analyzed using Cellquest 6.0 (BD Biosciences, NJ, USA) [15].

\section{Cell transfection}

In brief, the PC-3 cells seeded in a 6-well, 12-well, or 96-well plate were transfected using Lipofectamine 3000 transfection reagent (Invitrogen, CA, USA) according to the manufacturer's protocol. The following plasmids were used: pcDNA3-C/EBPa (a gift from Dr. Hong Yin), pcDNA3.1-2xFlag-SREBP-1a, and pcDNA3.1-2xFlag-SREBP-1c (from Addgene). The siRNA against C/EBPa (in short, Si-C/EBPa) and the negative control siRNA (in short, Si-Control) were designed and synthesized by RiboBio Company (Guangzhou, China). The siRNA against C/EBPa used in the present study was as follows: sense: 5'GGAGCUGACCAGUGACAAUdTdT-3'; and antisense: 5'-AUUGUCACUGGUCAGCUCCdTdT-3'.

Quantitative real-time PCR

Total RNA was extracted from PC-3 cells after transfection with C/EBPa-expressing plasmid or Si-C/EBPa and/or treatment with VPA (2.0 mM) using an E.Z.N.A. HP Total RNA Extraction Kit (Omega Bio-Tek, GA, USA). Reverse transcription reactions were performed with $2.0 \mu \mathrm{g}$ total RNA using a RevertAid First Strand cDNA Synthesis Kit and oligo (dT) primers (Fermentas Inc., Canada) according to the manufacturer's protocol. The abundance of mRNA was detected using an SYBR Green real-time PCR kit according to the manufacturer's instructions (Sangon Biotech, Shanghai, China). The transcript quantities of SREBP-1a and SREBP-1c were normalized to those of $\beta$-Actin and measured by the comparative $\mathrm{Ct}\left(2^{-\Delta \Delta C t}\right)$ method. The measurement was performed in three independent experiments and each with three replicates. The sequences of the primer pairs were as follows: SREBP-1a: 5'-CGGCGCTGCTGACCGACATC-3' and 5'CCCTGCCCCACTCCCAGCAT-3'; SREBP-1c: 5'-GCGCAGATCGCGGAGCCAT-3' and 5'- 
CCCTGCCCCACTCCCAGCAT-3'; and ß-Actin: 5'-CCAGAGATGGCCACGGCTGCT-3' and 5'TCCTTCTGCATCCTGTCGGCA-3'.

Statistical analysis

The experiments were repeated at least three times, and data were analyzed using SPSS (version 17.0; SPSS, Inc., IL, USA). All data were expressed as the mean \pm SD. A statistical analysis between two groups was performed using the Student's t test. One-way analysis of variance was used for the bar graphs containing three or more groups. ${ }^{*} \mathrm{P}<0.05,{ }^{* *} \mathrm{P}<0.01$, and ${ }^{\star \star *} \mathrm{P}<0.001$ indicate significant differences, whereas the nonsignificant difference is denoted by NS.

\section{Results}

The effect of VPA on PC-3 cell viability

The incidence of PCa presents an increasing trend in recent years. Previous studies indicated that VPA was a potential suppressor of the growth of PCa cells $[10,13,16]$. To test whether the growth inhibition was dose dependent, the PC-3 cells were treated with different concentrations of VPA (ranging from 0.5 to $10.0 \mathrm{mM}$ ) for 4 days, followed by the MTT cell viability assay. Our results showed that the growth of PC-3 cells was slow after VPA administration. The growth ability decreased in a dose-dependent manner, ranging from $7.52 \%$ for the low VPA concentration $(0.5 \mathrm{mM})$ up to $76.7 \%$ for the high VPA concentration $(10.0 \mathrm{mM})$ compared with the control cells (Fig. 1a, left). Meanwhile, PC-3 cells were treated with $2.0 \mathrm{mM}$ VPA over a time course of 1, 2, 4, and 6 days. As shown in Fig. 1a (right), VPA inhibited the growth of PC-3 cells significantly in a time-dependent manner, ranging from $9.73 \%$ on day 1 up to $64.4 \%$ on day 6 compared with the control cells. The inhibitory effect of VPA on PC-3 cell growth was further confirmed by the wound-healing assay. Consistently, similar results were observed, and showed that VPA markedly delayed the wound closure of PC-3 cells (Fig. 1b and 1c). Together, the results demonstrate that VPA remarkably prevents the $\mathrm{PC}-3$ cell viability.

The effect of VPA on lipogenesis in PC-3 cell

Cancer is a disorder characterized by increased metabolic activity, leading to enhanced cell growth and proliferation. Alterations in lipid metabolism are one of the main features in cancer cells $[5,17,18]$. Therefore, the functional effect of VPA on lipogenesis and apoptosis in PC-3 cells were determined in this study. PC-3 cells were treated with $0,0.5,1.0,2.0,5.0$, and $10.0 \mathrm{mM}$ VPA for 4 days, followed by Oil-red 0 staining and Western blot analysis. The results showed that VPA decreased lipid deposition in PC-3 cells in a dose-dependent manner, as evidenced by Oil-red 0 staining (Fig. 2a, b). The expression levels of FASN and ACC1, the key enzymes of de novo lipogenesis, were significantly suppressed in a dosedependent manner when PC-3 cells were cultured in a medium containing VPA (Fig. 2c, d).

The effect of VPA on apoptosis in PC-3 cell 
VPA is now a promising anticancer compound due to its pharmacological action on lipogenesis and apoptosis [8-10]. Therefore, the degree of apoptosis for VPA-treated PC-3 cells was analyzed by DAPI staining assay and Annexin V-PI staining assay. DAPI staining showed that the nuclear morphology of VPA-treated cells presented more and brighter blue fluorescence, including the rippled surface of nuclei, chromatin condensation, and nuclear fragmentation, compared with the control cells, suggesting the typical characteristics of apoptosis (Fig. 3a). To further investigate the apoptosis of PC-3 cells induced by VPA, the apoptotic cells were quantified with a flow cytometer using Annexin V-FITC and PI doublestaining. As shown in Fig. 3b and 3c, the percentage of apoptotic cells treated with VPA exhibited a significant rise compared with the control cells (from 8.89-22.73\%). Consistent with these results, Western blot analysis showed that the expression level of Bcl-2 in VPA-treated PC-3 cells significantly decreased in a dose-dependent manner (Fig. 3d, e). Collectively, the results above suggest that VPA suppresses the growth of PC-3 cells by inhibiting lipogenesis and inducing apoptosis.

C/EBPa regulated lipogenesis and apoptosis-related genes in VPA-treated PC-3 cells

C/EBPa and SREBP-1 play a pivotal role in lipid metabolism by inducing the transcription of genes related to lipogenesis $[8,19]$. Recent studies have revealed that C/EBPa and SREBP-1 are markedly upregulated in human cancers, providing the mechanistic link between lipid metabolism alterations and malignancies $[18,20,21]$. To identify the metabolic events occurring during VPA-induced antitumor progression, the expression levels of C/EBPa and SREBP-1 were determined by Western blot analysis. As shown in Fig. 4a and $4 \mathrm{~b}$, the expression levels of C/EBPa and SREBP-1 were significantly suppressed in PC-3 cells treated with VPA in a dose-dependent manner. Furthermore, the ectopic expression of C/EBPa inverted the downregulation of SREBP-1, FASN, ACC1, and Bcl-2 caused by VPA, as shown by Western blot analysis (Fig. 4c, d). The siRNA strategy was used to specifically knockdown C/EBPa. The Western blot analysis results confirmed the dramatic C/EBPa downregulation in PC-3 cells. As expected, decreasing the protein levels of SREBP-1, FASN, ACC1, and Bcl-2 were enhanced in PC-3 cells transfected with siRNA sequences specifically against $\mathrm{C} / \mathrm{EBPa}$ (in short, Si-C/EBPa) compared with the cells transfected with scrambled control siRNA (in short, Si-Control) (Fig. 4e, f). These results indicate that C/EBPa play an important role in expression of SREBP-1, FASN, ACC1, and Bcl-2 in VPA-treated PC-3 cells. Hence, it is presumed that the $\mathrm{C} / \mathrm{EBPa}$ /SREBP-1 pathway might be involved in the suppression of lipogenesis and induction of apoptosis in PC-3 cells after VPA administration.

Effects of SREBP-1a and SREBP-1c on expression of lipogenesis and apoptosis-related genes in VPAtreated PC-3 cells

The changes in cell metabolism and growth are closely linked through SREBPs. The SREBP family consists of three subtypes: SREBP-1a, SREBP-1c, and SREBP-2. SREBP-1a and SREBP-1c are derived from a single gene through the use of alternative transcription start sites. In general, SREBP-1c seems to act more specifically on the genes involved in fatty acid synthesis, while SREBP-1a has an influence on a large number of genes involved in the regulation of lipid metabolism, cell proliferation, differentiation, and death [19-22]. Importantly, our previous study indicated that C/EBPa enhanced SREBP-1a activation at 
the transcriptional level [23]. Hence, the present study examined the effect of C/EBPa on the transcriptional levels of SREBP-1a and SREBP-1c in PC-3 cells in the presence of VPA. PC-3 cells were transfected with C/EBPa-expressing plasmid or Si-C/EBPa for $48 \mathrm{~h}$ in the presence of VPA $(2.0 \mathrm{mM})$, and the transcriptional levels of SREBP-1a and SREBP-1c were tested by qRT-PCR analysis. The results demonstrated that ectopic expression of C/EBPa rescued the suppression of SREBP-1a and SREBP-1C activation, while Si-C/EBPa exerted an enhanced inhibitory effects in PC-3 cells exposed to VPA (Fig. 5a). In addition, the ectopic expression of SREBP-1a rescued the expression of FASN and ACC1, as well as the expression of Bcl-2 in VPA-treated cells (Fig. 5b, c). Differently from SREBP-1a, the ectopic expression of SREBP-1C rescued the expression of FASN and ACC1, but had no effect on Bcl-2 expression in VPAtreated cells (Fig. 5d, e).

Effects of C/EBPa/SREBP-1 pathway on lipogenesis and apoptosis in VPA-treated PC-3 cells

Subsequently, the effects of C/EBPa and SREBP-1 on PC-3 cell viability, lipogenesis, and apoptosis were examined. MTT cell viability assay showed an apparent increase in C/EBPa- or SREBP-1-expressing cells and a sharp decrease in Si-C/EBPa cells compared with the control cells (Fig. 6a). The ectopic expression of C/EBPa or SREBP-1 led to a significant increase in lipid accumulation in PC-3 cells treated with VPA compared with the control cells. The loss of lipids and smaller lipid droplets as a result of $\mathrm{C} / \mathrm{EBPa}$ knockdown was also confirmed by Oil-red 0 staining (Fig. 6b, c). Enhancing C/EBPa or SREBP-1 expression partially suppressed and Si-C/EBPa enhanced VPA-induced apoptosis in PC-3 cells (Fig. 6d, e). Taken together, it is concluded that VPA treatment significantly decreases lipid accumulation and induces apoptosis as a result of the inhibition of C/EBPa, as well as SREBP-1 and its target gene expression. VPAtriggered suppression of C/EBPa/SREBP-1 pathway might provide mechanistic insights and offer a novel therapeutic strategy for $\mathrm{PCa}$.

\section{Discussion}

Metabolic reprogramming not only promotes cancer cell plasticity but also provides novel insights for treatment strategies. Altered lipid metabolism is increasingly recognized as a signature of cancer cells, and the proteins involved in this process can be excellent chemotherapeutic targets for cancer treatment [24-27]. A link between PCa progression and lipogenesis has been investigated in the last few decades. Several recent studies suggest that the activation of de novo lipogenesis and cholesterogenesis induces PCa cell proliferation and promotes PCa development and progression. Hence, targeting the lipogenesis pathway may be a promising avenue for PCa treatment [26-29].

VPA is known as a histone deacetylase inhibitor and an anticonvulsant and mood-stabilizing drug. It has been reported as a potent and promising anticancer drug candidate [10-12]. In the present study, the anticancer effects of VPA on human prostate cancer PC-3 cells were detected using MTT and woundhealing assay. The results indicated that VPA inhibited cell growth significantly in a dose-dependent and time-dependent manner. Moreover, VPA significantly inhibited lipogenesis and induced cellular apoptosis in PC-3 cells. Further assays were performed to analyze the underlying molecular mechanisms. C/EBPa 
and SREBP-1 are crucial factors controlling lipogenesis. It is reported that VPA suppresses the accumulation of intracellular triacylglycerol and decreases the expression level of C/EBPa [8]. In addition, a growing amount of evidence suggests that aberrant SREBP-1 activity can contribute to cancer [30]. A previous study indicated that SREBP-1 was overexpressed and involved in the transcriptional regulation of fatty acid synthesis through the altered expression of FASN in prostate cancer cells [19]. Hence, the present study concentrated on C/EBPa and SREBP-1. Our results showed that the expression levels of C/EBPa and SREBP-1, as well as their target genes FASN, ACC1, and Bcl-2, decreased significantly after VPA treatment.

An adapted "metabolic switch" accompanies most physiological and pathological changes in cellular functions. Lipid metabolism is essential for cancer cells and is associated with the regulation of a variety of key cellular processes and functions [31-33]. The cellular metabolites can directly or indirectly regulate the apoptotic machinery. Metabolism is emerging as one of the key factors contributing to the evasion of apoptosis in cancer [34]. The suppression of C/EBPa inhibits cell proliferation by inducing G1phase arrest and apoptosis [20]. Emerging evidence indicates that SREBP-1a couples lipid synthesis to cell progression and apoptosis. A recent study shows that SREBP-1a regulates antiapoptotic factor apoptosis inhibitor 6 (Api6) besides regulating lipid metabolism in response to nutrient levels [28, 35]. Consistently, our results revealed that the antiapoptotic Bcl-2 expression regulated by SREBP-1a might be involved in the apoptosis of VPA-treated PC-3 cells. Our study suggests distinct roles of the SREBP-1 isoforms. SREBP-1a might play a more important role in VPA-induced apoptosis in PC-3 cells.

\section{Conclusions}

Together, the present study indicate that VPA inhibits prostate cancer growth via the C/EBPa/SREBP-1 pathway targeting lipogenesis and apoptosis. Therefore, blocking fatty acid biosynthesis could be a potential treatment for $\mathrm{PCa}$.

\section{Abbreviations}

ACC1:acetyl-coenzyme A carboxylase 1; Bcl-2:antiapoptotic B cell lymphoma 2; Api6:antiapoptotic factor apoptosis inhibitor 6; C/EBPa:CCAAT/enhancer-binding protein a;DAPI:4', 6'-diamidino-2-phenyl-indole; FASN:fatty acid synthase; FITC:fluorescein isothiocyanate; MTT:3-(4, 5-dimethylthiazol-2-yl)-2, 5diphenyltetrazolium bromide; OD:optical density; PBS:phosphate-buffered saline; PI:propidium iodide; PCa:prostate cancer; qRT-PCR:quantitative real-time PCR; SREBP-1:sterol regulatory element-binding protein 1; SREBP-1a:sterol regulatory element-binding protein-1a; SREBP-1c:sterol regulatory elementbinding protein-1c; VPA:valproic acid

\section{Declarations}

\section{Acknowledgements}


We would like to thank MedSci for reviewing and providing professional editing services for the manuscript.

\section{Authors' contributions}

BP performed experiments, analyzed data and helped write the manuscript. JZ, JY and YS helped to design and perform experiments. LQ conceived the project, designed the overall research, wrote the manuscript, mentored and supervised participants. All authors have read, commented on, and approved the final manuscript.

\section{Funding}

This study was supported by the National Natural Science Foundation of China $(81370955,81300664$, and 81800767) and the Tianjin Natural Science Foundation (18JCYBJC25500).

\section{Availability of data and materials}

The authors confirm that all materials described in the manuscript are fully available to any scientist wishing to use them, without restriction.

\section{Ethics approval}

Not applicable.

\section{Consent for publication}

All authors agree to publish this article in the journal of Lipids in Health and Disease.

\section{Competing interests}

The authors declare that they have no competing interests

\section{Author details}

NHC Key Laboratory of Hormones and Development (Tianjin Medical University), Tianjin Key Laboratory of Metabolic Diseases, Tianjin Medical University Chu Hsien-I Memorial Hospital \& Tianjin Institute of Endocrinology, Tianjin Medical University, Tianjin 300134, China.

\section{References}

1. Nickels JT Jr. New links between lipid accumulation and cancer progression. J Biol Chem. 2018; 293 (17): 6635-6636.

2. Guri Y, Colombi M, Dazert E, et al. mTORC2 Promotes Tumorigenesis via Lipid Synthesis. Cancer Cell. 2017; 32(6): 807-823. 
3. Gordon JA, Noble JW, Midha A, et al. Upregulation of Scavenger Receptor B1 Is Required for Steroidogenic and Nonsteroidogenic Cholesterol Metabolism in Prostate Cancer. Cancer Res. 2019; 79(13): 3320-3331.

4. Yue S, Li J, Lee SY, et al. Cholesteryl ester accumulation induced by PTEN loss and PI3K/AKT activation underlies human prostate cancer aggressiveness. Cell Metab. 2014; 9(3): 393-406.

5. Singh KB, Kim SH, Hahm ER, et al. Prostate cancer chemoprevention by sulforaphane in a preclinical mouse model is associated with inhibition of fatty acid metabolism. Carcinogenesis. 2018; 39(6): 826-837.

6. Zadra G, Ribeiro CF, Chetta $P$, et al. Inhibition of de novo lipogenesis targets androgen receptor signaling in castration-resistant prostate cancer. Proc Natl Acad Sci U S A. 2019; 116(2): 631-640.

7. Penfold L, Woods A, Muckett P, et al. CAMKK2 Promotes Prostate Cancer Independently of AMPK via Increased Lipogenesis. Cancer Res. 2018; 78(24): 6747-6761.

8. Yuyama M, Fujimori K. Suppression of adipogenesis by valproic acid through repression of USF1activated fatty acid synthesis in adipocytes. Biochem J. 2014; 459(3): 489-503.

9. Avery LB, Bumpus NN. Valproic acid is a novel activator of AMP-activated protein kinase and decreases liver mass, hepatic fat accumulation, and serum glucose in obese mice. Mol Pharmacol. 2014; 85(1): 1-10.

10. Kawashima N, Nishimiya Y, Takahata S, et al. Induction of Glycosphingolipid GM3 Expression by Valproic Acid Suppresses Cancer Cell Growth. J Biol Chem. 2016; 291(41): 21424-21433.

11. Zapotocky M, Mejstrikova E, Smetana K, et al. Valproic acid triggers differentiation and apoptosis in AML1/ETO-positive leukemic cells specifically. Cancer Lett. 2012; 319(2): 144-153.

12. Roos WP, Jöst E, Belohlavek $C$, et al. Intrinsic anticancer drug resistance of malignant melanoma cells is abrogated by IFN- $\beta$ and valproic acid. Cancer Res. 2011; 71(12): 4150-60.

13. Makarević J, Rutz J, Juengel E, et al. HDAC Inhibition Counteracts Metastatic Re-Activation of Prostate Cancer Cells Induced by Chronic mTOR Suppression. Cells. 2018; 7(9): pii: E129.

14. Liu L, Gao H, Wang H, et al. Catalpol promotes cellular apoptosis in human HCT116 colorectal cancer cells via microRNA-200 and the downregulation of PI3K-Akt signaling pathway. Oncol Lett. 2017; 14(3): 3741-3747.

15. Wang, H. Li, X. Wang, et al. Alisol B-23-acetate, a tetracyclic triterpenoid isolated from Alisma orientale, induces apoptosis in human lung cancer cells via the mitochondrial pathway. Biochem Biophys Res Commun. 2018; 505(4): 1015-1021.

16. Chou YW, Chaturvedi NK, Ouyang S, et al. Histone deacetylase inhibitor valproic acid suppresses the growth and increases the androgen responsiveness of prostate cancer cells. Cancer Lett. 2011; 311(2): 177-86.

17. Xie H, Simon MC. Oxygen availability and metabolic reprogramming in cancer. J Biol Chem. (2017); 292(41): 16825-16832. 
18. Guo D, Reinitz F, Youssef M, et al. An LXR agonist promotes glioblastoma cell death through inhibition of an EGFR/AKT/SREBP-1/LDLR-dependent pathway. Cancer Discov. 2011; 1(5): 442-56.

19. Walker AK, Jacobs RL, Watts JL, et al. A conserved SREBP-1/phosphatidylcholine feedback circuit regulates lipogenesis in metazoans. Cell. 2011; 147(4): 840-52.

20. Lee J, Imm JY, Lee SH. $\beta$-Catenin Mediates Anti-adipogenic and Anticancer Effects of Arctigenin in Preadipocytes and Breast Cancer Cells. J Agric Food Chem. 2017; 65(12): 2513-2520.

21. Li W, Tai Y, Zhou J, et al. Repression of endometrial tumor growth by targeting SREBP1 and lipogenesis. Cell Cycle. 2012; 11(12): 2348-58.

22. Shao W, Espenshade PJ. Expanding roles for SREBP in metabolism. Cell Metab. 2012; 16(4): 414419.

23. Qiao $L$, Wu Q, Lu X, et al. SREBP-1a activation by HBx and the effect on hepatitis $B$ virus enhancer II/core promoter. Biochem Biophys Res Commun. 2013; 432(4): 643-649.

24. Li, M. Li, J. Hu, et al., The microRNA-182-PDK4 axis regulates lung tumorigenesis by modulating pyruvate dehydrogenase and lipogenesis. Oncogene. 2017; 36(7): 989-998.

25. Gomes AS, Ramos H, Soares J, et al. p53 and glucose metabolism: an orchestra to be directed in cancer therapy. Pharmacol Res. 2018; 131: 75-86.

26. Zadra G, Photopoulos C, Tyekucheva S, et al. A novel direct activator of AMPK inhibits prostate cancer growth by blocking lipogenesis. EMBO Mol Med. 2014; 6(4): 519-38.

27. Dasgupta S, Putluri N, Long W, et al. Coactivator SRC-2-dependent metabolic reprogramming mediates prostate cancer survival and metastasis. J Clin Invest. 2015; 125(3): 1174-88.

28. O'Malley J, Kumar R, Kuzmin AN, et al. Lipid quantification by Raman microspectroscopy as a potential biomarker in prostate cancer. Cancer Lett. 2017; 397: 52-60.

29. Chen J, Guccini I, Mitri DD, et al. Compartmentalized activities of the pyruvate dehydrogenase complex sustain lipogenesis in prostate cancer. Nat Genet. 2018; 50(2): 219-228.

30. Talebi A, Dehairs J, Rambow F, et al. Sustained SREBP-1-dependent lipogenesis as a key mediator of resistance to BRAF-targeted therapy. Nat Commun. 2018; 9(1): 2500.

31. Iwamoto H, Abe M, Yang Y, et al. Cancer Lipid Metabolism Confers Antiangiogenic Drug Resistance. Cell Metab. 2018; 28(1): 104-117.

32. Stockwell BR, Friedmann Angeli JP, Bayir H, et al. Ferroptosis: A Regulated Cell Death Nexus Linking Metabolism, Redox Biology, and Disease. Cell. 2017; 171(2): 273-285.

33. Pommier AJ, Alves G, Viennois E, et al. Liver X Receptor activation downregulates AKT survival signaling in lipid rafts and induces apoptosis of prostate cancer cells. Oncogene. 2010; 29(18): 271223.

34. Matsuura K Canfield K, Feng W, et al., Metabolic Regulation of Apoptosis in Cancer. Int Rev Cell Mol Biol. 2016; 327: 43-87.

35. Im SS, Osborne TF. Protection from bacterial-toxin-induced apoptosis in macrophages requires the lipogenic transcription factor sterol regulatory element binding protein 1a. Mol Cell Biol. 2012; 


\section{Figures}

A

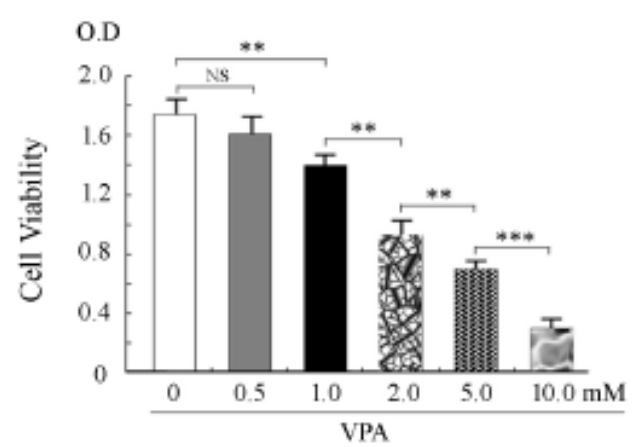

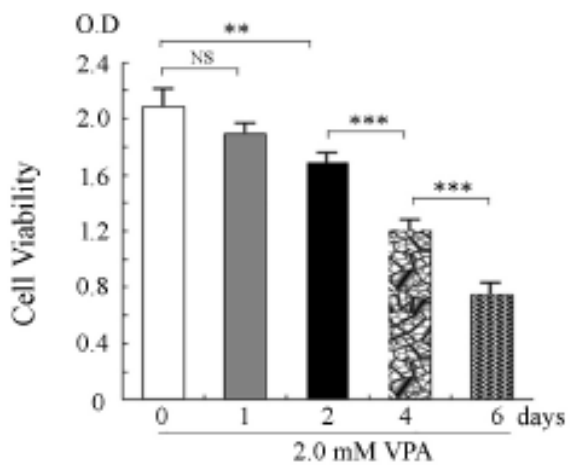

B
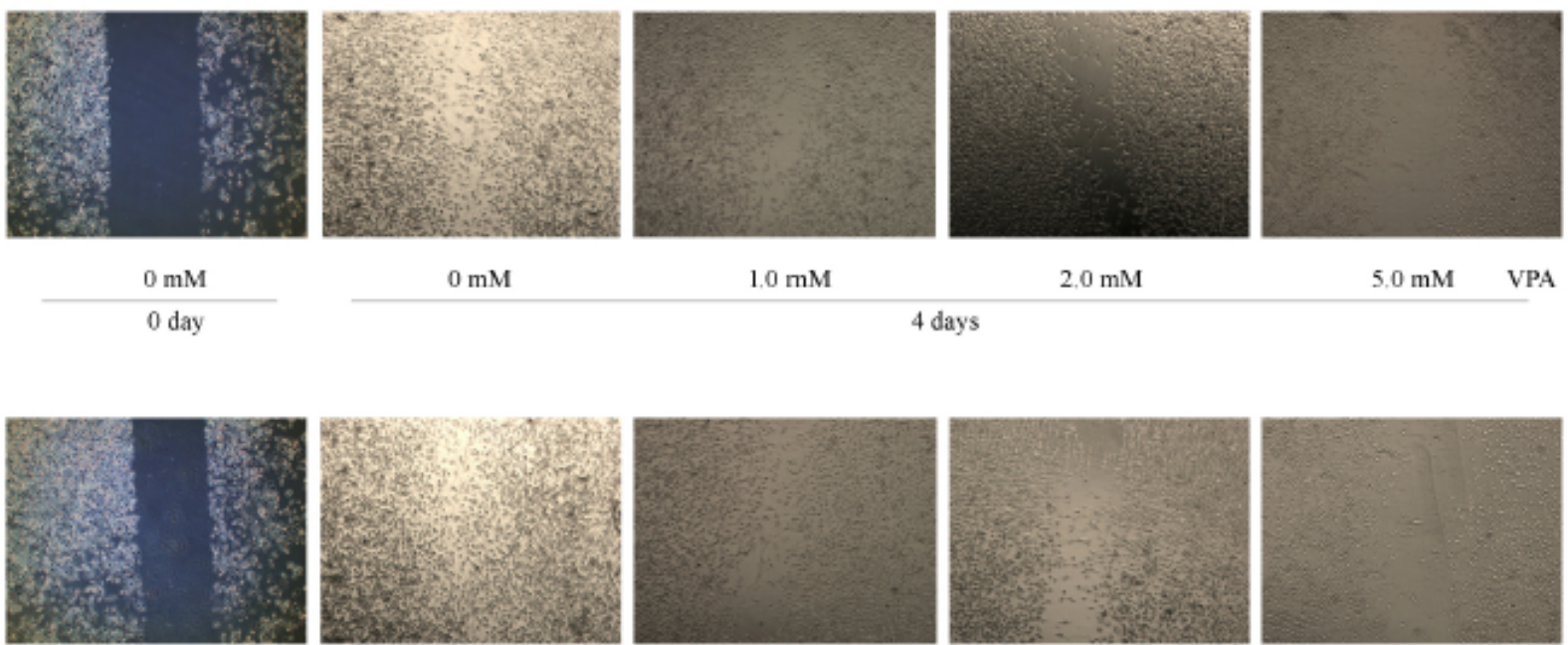

$1.0 \mathrm{mM}$

$2,0 \mathrm{mM}$

$5.0 \mathrm{mM} \quad$ VPA
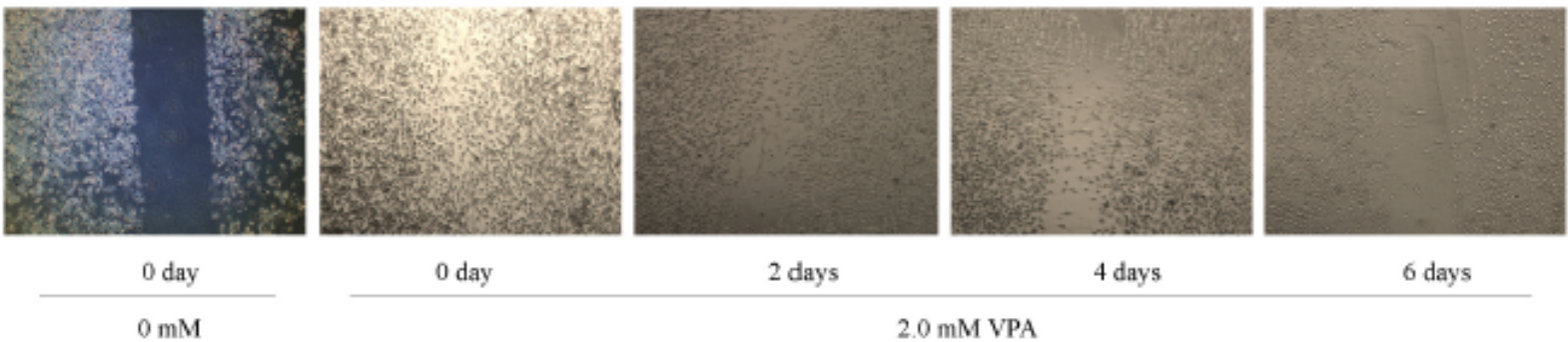

2 days

4 days

6 days

$2.0 \mathrm{mM}$ VPA

C
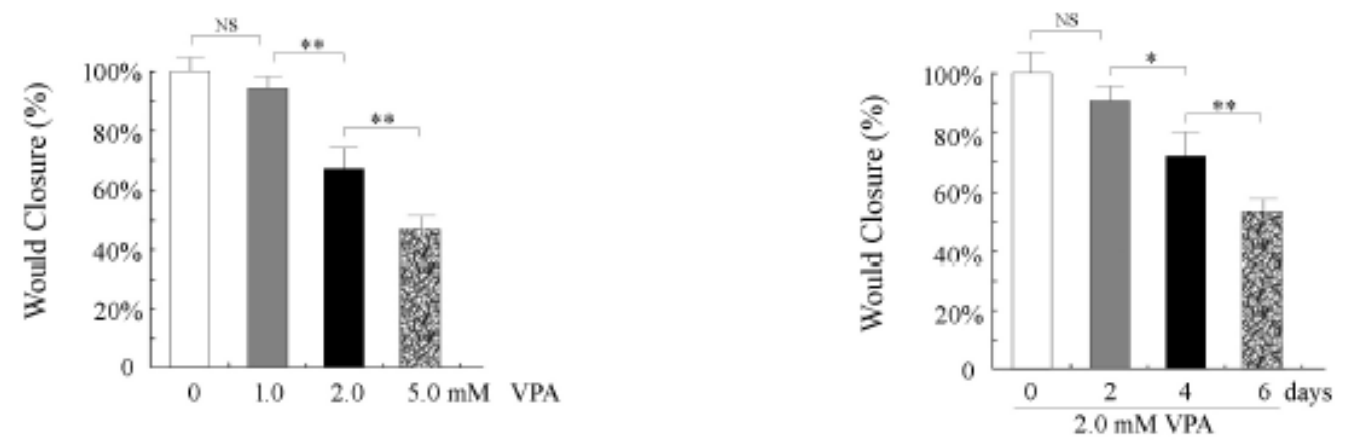

Figure 1 
Effects of VPA on PC-3 cell viability. (a) Viability of PC-3 cells after treatment with increasing concentrations of VPA $(0,0.5,1.0,2.0,5.0$, and $10.0 \mathrm{mM})$ for 4 days was measured using the MTT assay. VPA significantly inhibited cell growth in a dose-dependent manner (left). The viability of PC-3 cells with VPA treatment $(2.0 \mathrm{mM})$ was measured at various time points $(0,1,2,4$, and 6 days). VPA significantly inhibited cell growth in a time-dependent manner (right). (b) Representative PC-3 cell wound healing width images. The migration of PC-3 cells treated with various concentrations of VPA for 4 days (up) or VPA $(2.0 \mathrm{mM}$ ) for $0,2,4$, and 6 days (down) was assessed using the wound-healing assay. (c) The PC-3 cell migration rate was calculated according to the width from 0 to 6 days. Data are expressed as mean $\pm S D$, $n=3$. Statistically significant differences are indicated: NS (no significance), $* P<0.05, \star \star P<0.01$, and $* * * P<0.001$.

$\dot{A}$

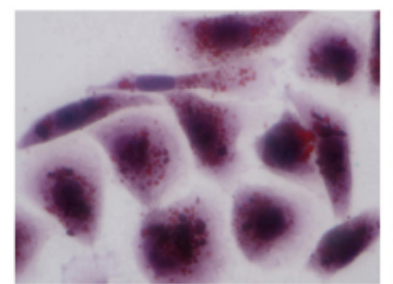

$0 \mathrm{mM}$

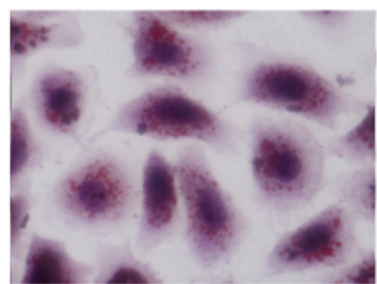

$0.5 \mathrm{mM}$

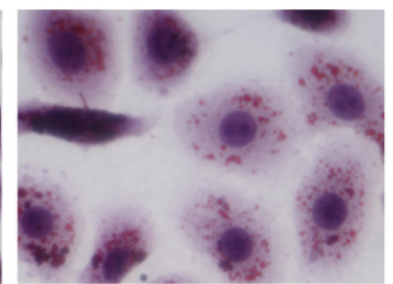

$1.0 \mathrm{mM}$

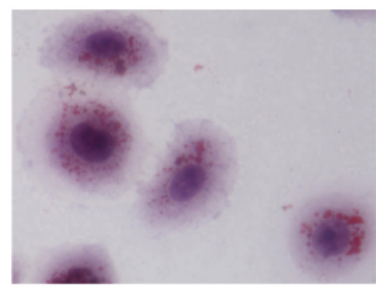

$2.0 \mathrm{mM}$

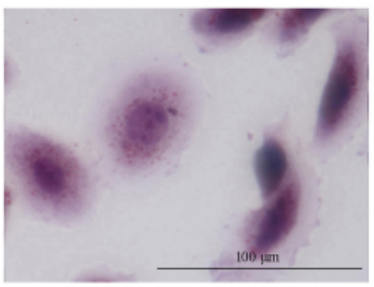

$5.0 \mathrm{mM}$ VPA
B

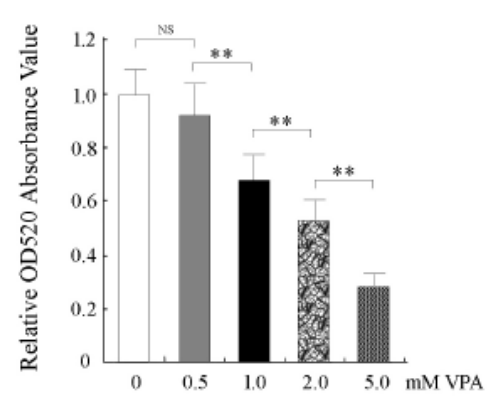

C

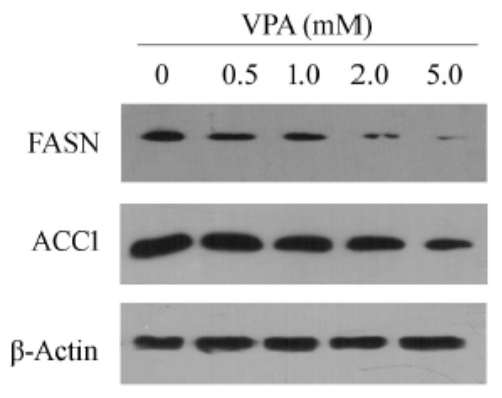

$\mathrm{D}$

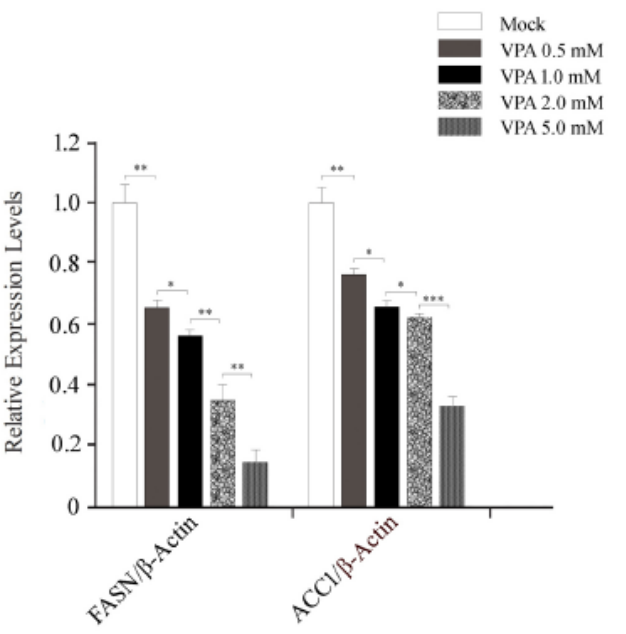

Figure 2

The effect of VPA on lipogenesis in PC-3 cell. (a) Effect of VPA on lipid storage in PC-3 cells was detected by Oil-Red $O$ staining. (b) Oil-Red O extracted with isopropanol was measured at OD520. Scale bar $=100$ $\mu \mathrm{m}$. (c) Western blot detection of the lipogenic gene expression of PC-3 cells after treatment with various concentrations of VPA. $\beta$-Actin was used as a loading control. (d) A semi-quantitative analysis of lipogenic gene expression was performed using ImageJ. 
A

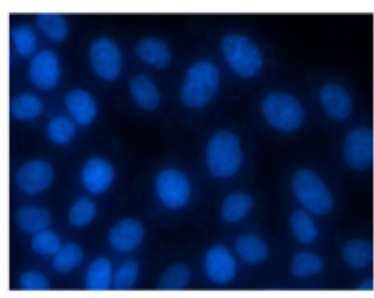

0

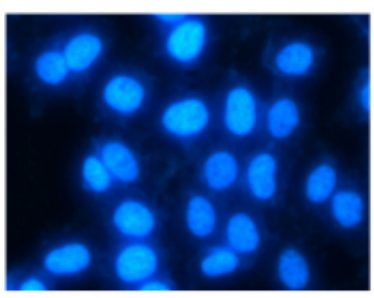

$1.0 \mathrm{mM}$

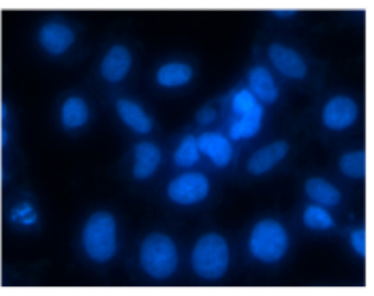

$2.0 \mathrm{mM}$

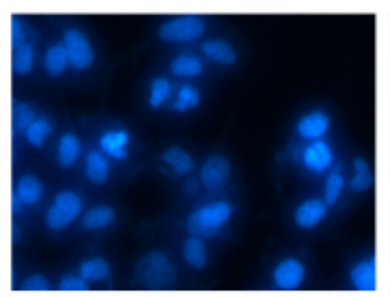

$5.0 \mathrm{mM}$

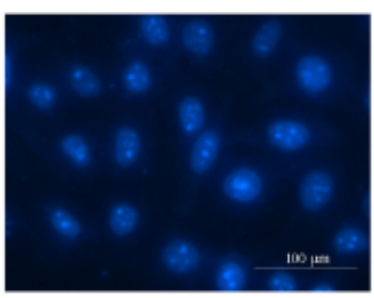

$10.0 \mathrm{mM}$ VPA

4 days

B
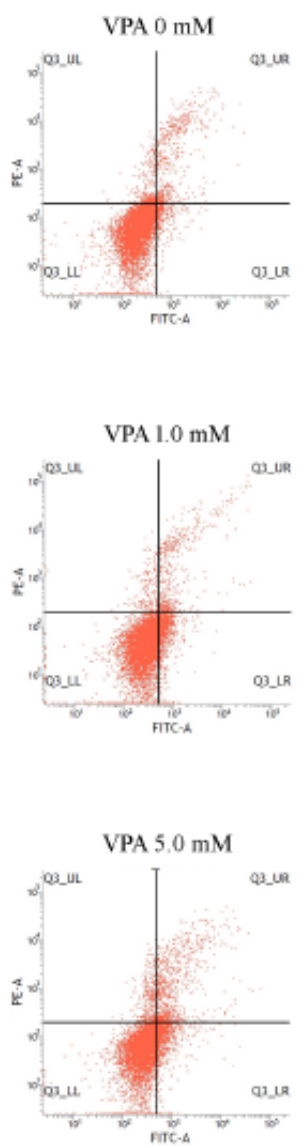

C

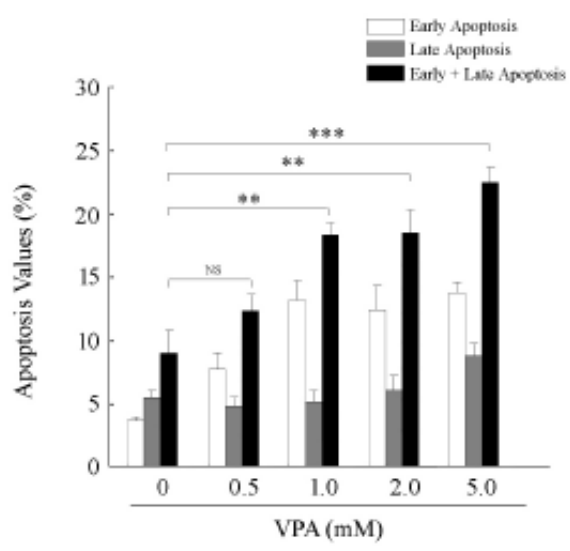

D

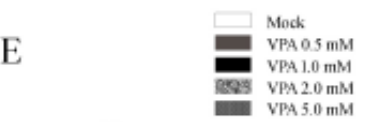

Bcl-2

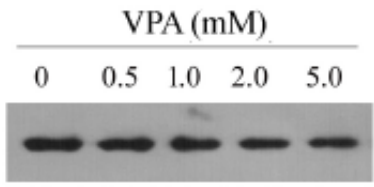

$\beta$-Actin
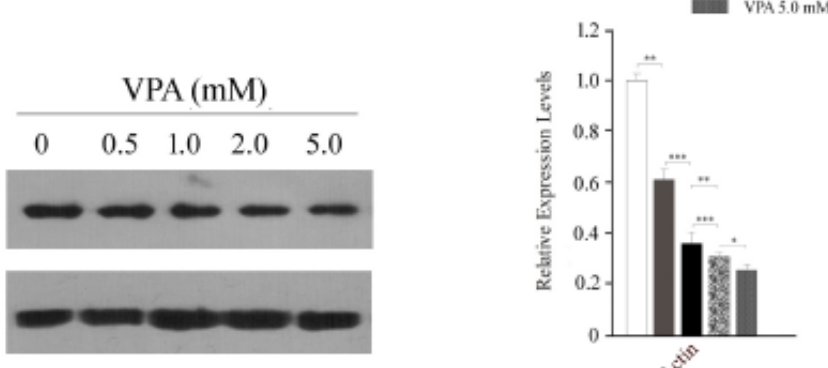

\section{Figure 3}

The effect of VPA on apoptosis in PC-3 cell. (a) PC-3 cells were incubated with various concentrations of VPA for 4 days, and the changes in nuclear morphology were evaluated using DAPI staining. (b) Effects of VPA on the apoptosis of PC-3 cells. PC-3 cells were treated with 0 (control), $0.5,1.0,2.0$, and $5.0 \mathrm{mM}$ VPA. The apoptotic rate was measured by flow cytometry with Annexin V-FITC/PI staining. PI, Propidium iodide. (c) Summary of the percentage of apoptotic cells treated with various concentrations of VPA. (d) Level of Bcl-2 in PC-3 cells was analyzed by Western blot analysis. $\beta$-Actin was used as a loading control. (e) The fold-change was calculated based on the densitometric analysis of the band intensities. All 
experiments were performed three times. Data are expressed as mean \pm SD. Statistically significant differences are indicated: NS (no significance), ${ }^{*} \mathrm{P}<0.05,{ }^{*} \mathrm{P}<0.01$, and $* \star * \mathrm{P}<0.001$.

A
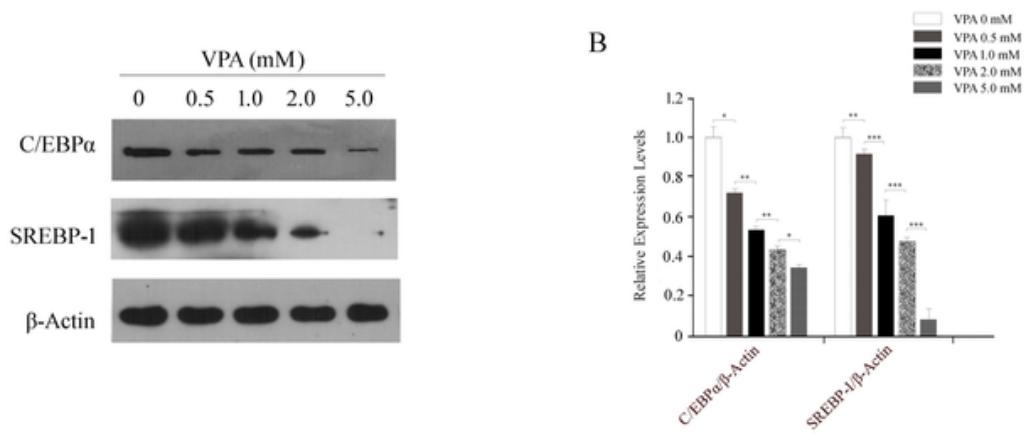

C

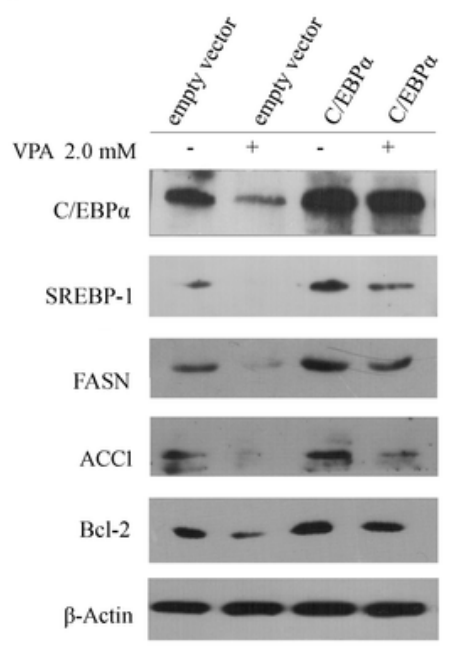

$\mathrm{D}$

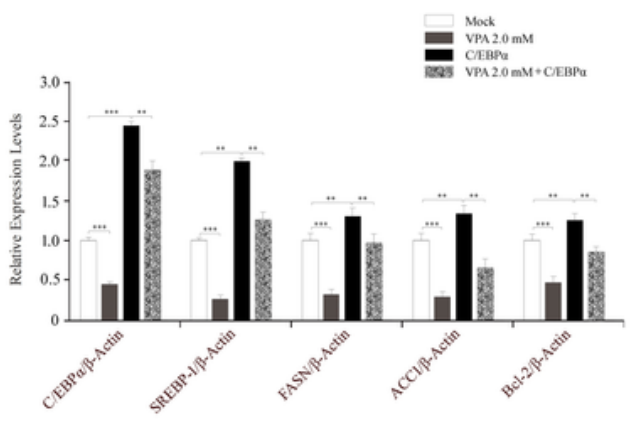

E

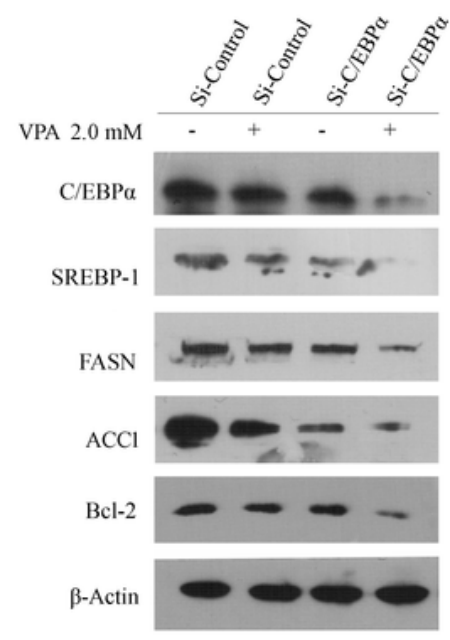

$\mathrm{F}$

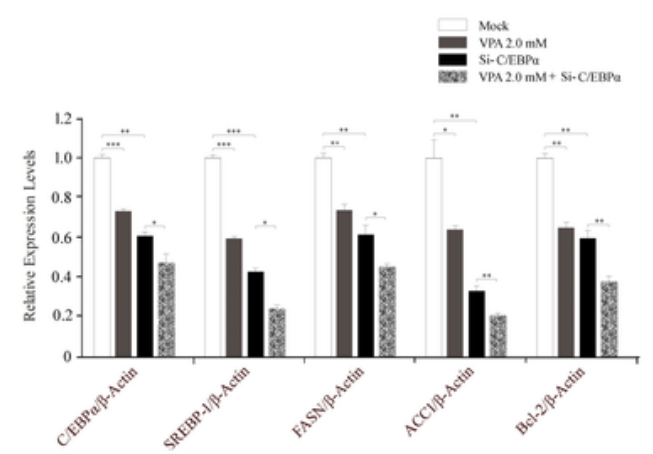

Figure 4

C/EBPa regulated lipogenesis and apoptosis-related genes in VPA-treated PC-3 cells ( $a$ and $b$ ) Expression of C/EBPa and SREBP-1 in PC-3 cells treated with various concentrations of VPA was analyzed by Western blot analysis. The levels of C/EBPa and SREBP-1 were quantified by densitometry and 
normalized with those of $\beta$-Actin. (c and d) PC-3 cells were transfected with C/EBPa-expressing plasmid, followed by treatment without or with $2.0 \mathrm{mM}$ VPA for $48 \mathrm{~h}$. The levels of C/EBPa, SREBP-1, FASN, ACC1, and $\mathrm{Bcl}-2$ were measured by Western blot analysis. The fold-change was calculated based on a densitometric analysis of the band intensities. Mock: The cells were transfected with empty plasmid. (e and f) PC-3 cells were transfected with Si-C/EBPa, followed by VPA treatment. The levels of C/EBPa, SREBP-1, FASN, ACC1, and BCl-2 were measured by Western blot analysis. The fold-change was calculated based on the densitometric analysis of the band intensities. Mock: The cells were transfected with scrambled control si-RNA (Si-Control). Statistically significant differences are indicated: NS (no significance), ${ }^{\star} \mathrm{P}<0.05, \star \star \mathrm{P}<0.01$, and ${ }^{\star \star *} \mathrm{P}<0.001$. 
A

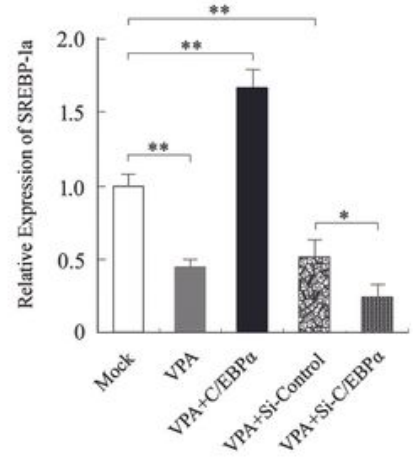

B

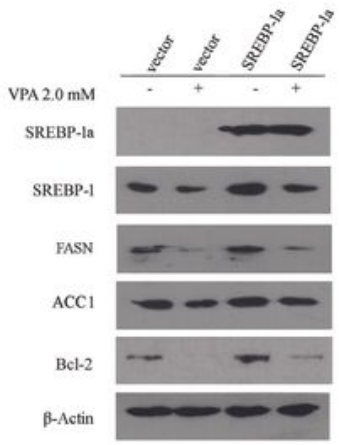

D

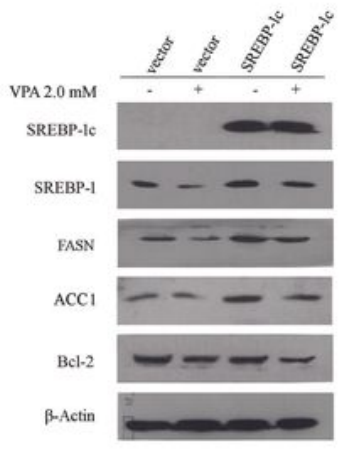

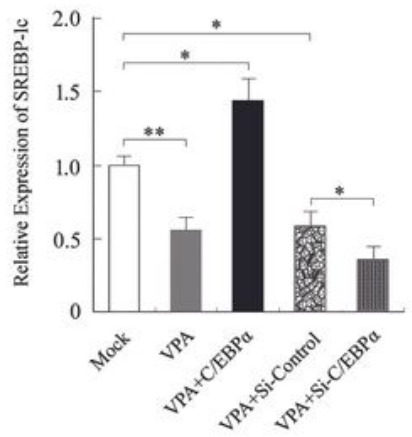

C

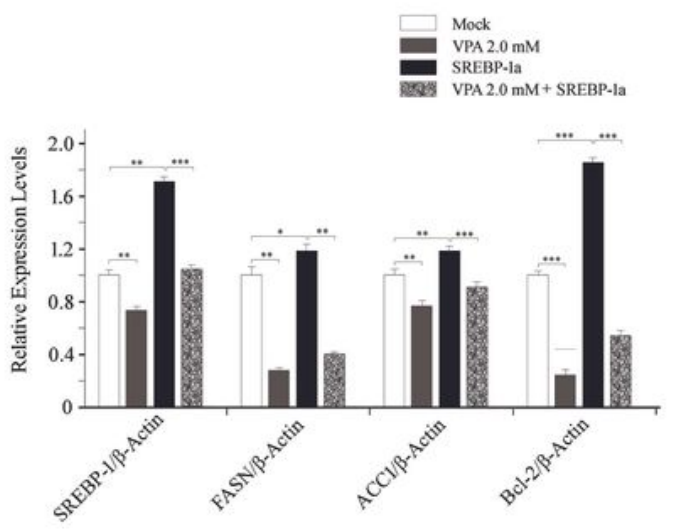

E

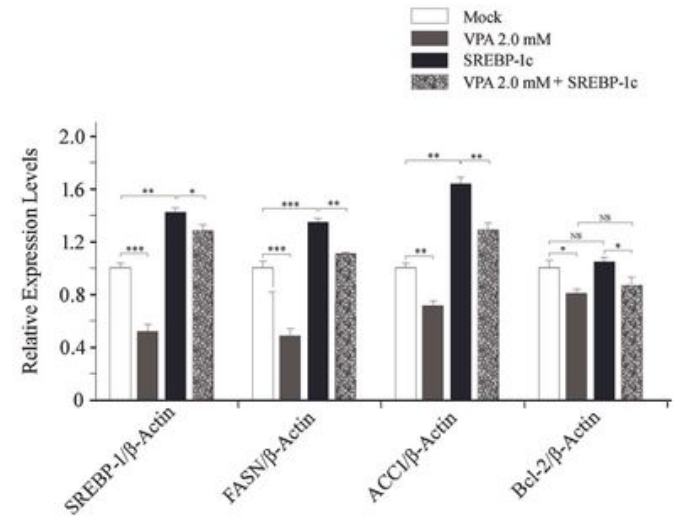

Figure 5

Effects of SREBP-1a and SREBP-1c on expression of lipogenesis and apoptosis-related genes in VPAtreated PC-3 cells (a) qRT-PCR analysis of the mRNA levels of SREBP-1a and 1c in PC-3 cells after transfection with C/EBPa or Si-C/EBPa and treatment without or with VPA. $\beta$-Actin was used for normalization. ( $b$ and $c$ ) Cells were transfected with SREBP-1a-expressing plasmid, followed by treatment without or with $2.0 \mathrm{mM}$ VPA. The protein expression in treated cells was determined by Western blot 
analysis. $\beta$-Actin was used as an internal reference. ( $d$ and e) Expression levels of SREBP-1, FASN, ACC1, and $\mathrm{Bcl}-2$ were analyzed by Western blot analysis in VPA-treated PC-3 cells after transfection with SREBP$1 \mathrm{c}$.

A

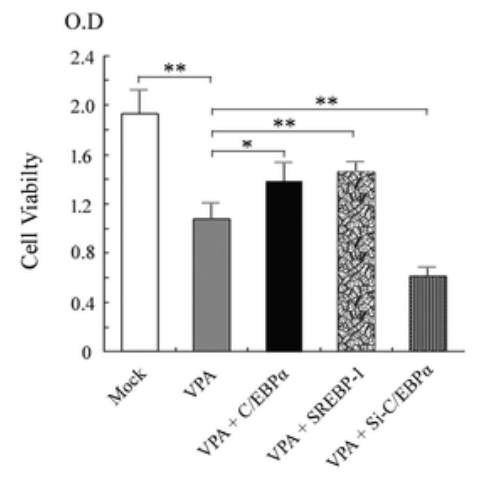

C

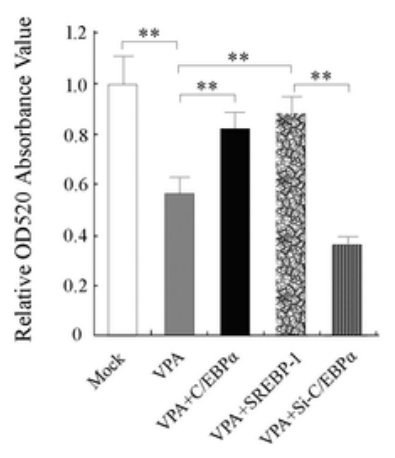

B

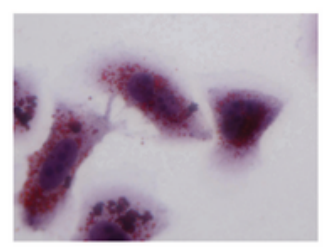

Mock

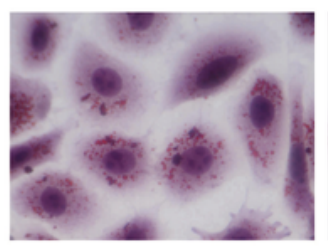

$2.0 \mathrm{mM}$ VPA

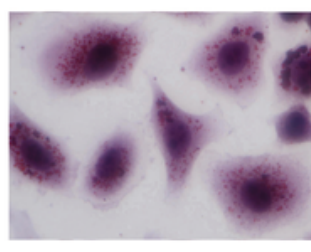

$\mathrm{VPA}+\mathrm{C} / \mathrm{EBP} \alpha$

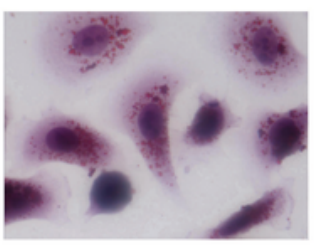

VPA + SREBP-1

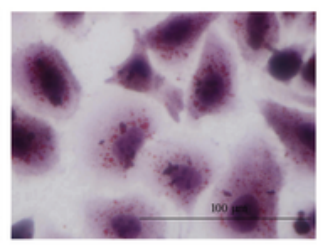

$\mathrm{VPA}+\mathrm{Si}-\mathrm{C} / \mathrm{EBP} \alpha$

D
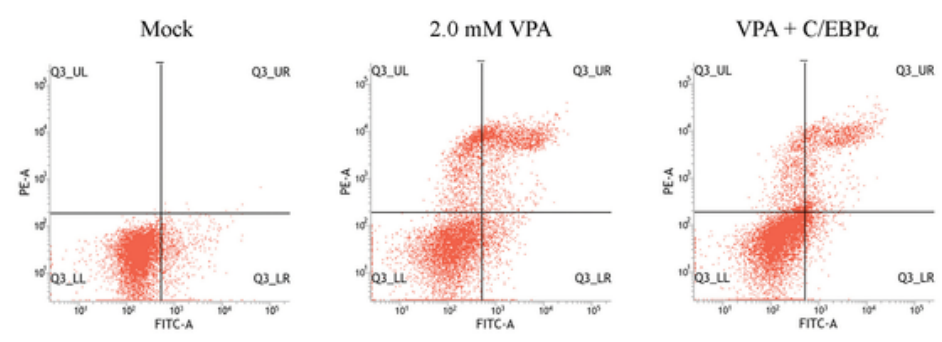

E
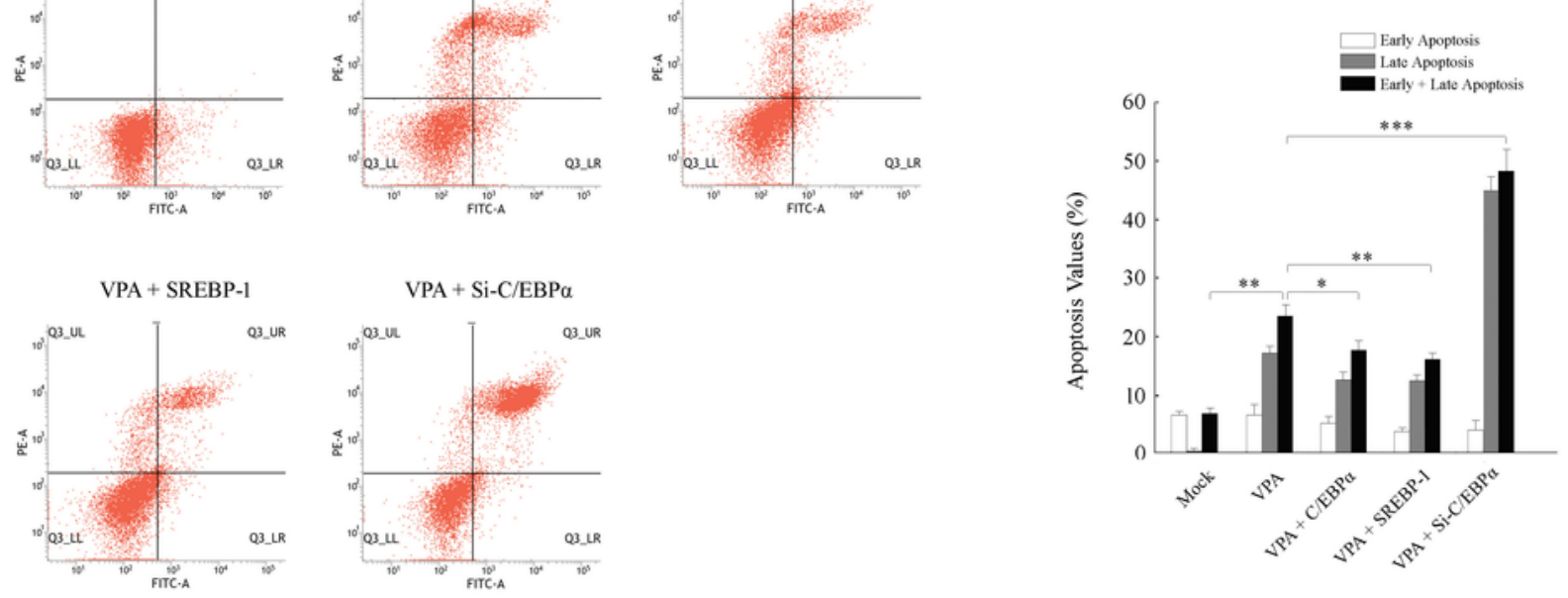

Figure 6

Effects of C/EBPa/SREBP-1 pathway on lipogenesis and apoptosis in VPA-treated PC-3 cells (a) PC-3 cells were transfected with an expressing plasmid encoding C/EBPa or SREBP-1a/c, Si-Control, or Si- 
$\mathrm{C} / \mathrm{EBPa}$, followed by treatment without or with $2.0 \mathrm{mM}$ VPA. The cell viability was determined using MTT assay. (b) Representative microscopic images of Oil-Red $O$ staining of PC-3 cells transfected with C/EBPa- or SREBP-1-expressing plasmid or Si-C/EBPa following treatment with VPA (2.0 mM) for $48 \mathrm{~h}$. (c) Oil-Red O extracted with isopropanol was measured at OD520. (d) Representative dot plots of flow cytometric cell viability measurement on PC-3 cells under different treatments. (e) Percentages of apoptotic cells were analyzed by flow cytometry. The values represent the mean \pm SD of three experiments. Statistically significant differences are indicated: NS (no significance), ${ }^{\star} P<0.05, \star \star P<0.01$, and $* \star * P<0.001$. 